\title{
Validação de um instrumento por meio de métodos da análise multivariada acerca das concepções do ensino de ciências do nono ano do ensino fundamental
}

\author{
Validation of an instrument using methods of multivariate analysis about the conceptions of science \\ teaching in the ninth grade of elementary school \\ Validación de un instrumento mediante métodos de análisis multivariado sobre los conceptos de \\ educación científica en el noveno grado de la escuela primaria
}

Recebido: 16/05/2021 | Revisado: 21/05/2021 | Aceito: 23/05/2021 | Publicado: 08/06/2021

\author{
Cíntia Moralles Camillo \\ ORCID: https://orcid.org/0000-0003-2876-9156 \\ Universidade Federal de Santa Maria, Brasil \\ E-mail: cintiacamillo@gmail.com \\ Karine Gehrke Graffunder \\ ORCID: https://orcid.org/0000-0001-8860-9889 \\ Universidade Federal de Santa Maria, Brasil \\ E-mail: ka.graffunder@gmail.com
}

\begin{abstract}
Resumo
O objetivo deste estudo é descrever o processo de construção e validação de um instrumento por meio da Análise Multivariada, no qual utilizou-se a técnica da Análise Fatorial combinada com a Análise de Cluster, a fim de analisar as concepções dos alunos do Ensino Médio sobre a importância da disciplina de Ciências do nono ano do Ensino Fundamental, com ênfase em conceitos de Física (Óptica). Para isso, aplicou-se um questionário piloto, disponibilizado de forma virtual para 21 alunos do Ensino Médio de um colégio público federal, localizado na cidade de Campo Grande do estado do Mato Grosso do Sul. Esta pesquisa é de cunho quantitativo, no qual se busca responder o objeto de estudo, caracterizando-se como conclusiva. A pesquisa requer informações definidas, formal e estruturadas, por isso se apresenta uma amostra e a análise dos dados são estatísticos e conclusivos. Ao aplicar-se todos os testes e análises (Alfa de Cronbach, Teste de Bartlett, KMO, Análise dos Componentes Principais, Análise Fatorial e Análise de Cluster), conclui-se que o instrumento possui confiabilidade, comunalidade, adequabilidade e interdependência entre as variáveis, sendo validado por técnicas da Análise Multivariada.
\end{abstract}

Palavras-chave: Ensino; Alfa de Cronbach; Análise fatorial; Análise de Cluster; Física.

\begin{abstract}
This study aimed to describe the process of building and validating an instrument through Multivariate Analysis, in which the Factor Analysis technique combined with Cluster Analysis was used, in order to analyze the conceptions of high school students about the importance of the Science discipline in the ninth year of Elementary School, with an emphasis on Physics concepts (Optics). To this end, a pilot questionnaire was applied, made available virtually to 21 high school students from a federal public college, located in the city of Campo Grande in the state of Mato Grosso do Sul. This research is of a quantitative nature, in which it seeks to answer the object of study, being characterized as conclusive. The research requires defined, formal and structured information, so a sample is presented and the data analysis is statistical and conclusive. When applying all tests and analyzes (Cronbach's Alpha, Bartlett's Test, KMO, Principal Component Analysis, Factor Analysis and Cluster Analysis), it is concluded that the instrument has reliability, commonality, suitability and interdependence between the variables, being validated by Multivariate Analysis techniques.
\end{abstract}

Keywords: Teaching; Cronbach's alpha; Factor analysis; Cluster Analysis; Physics.

\section{Resumen}

El objetivo de este estudio es describir el proceso de construcción y validación de un instrumento a través del Análisis Multivariado, en el cual se utilizó la técnica de Análisis Factorial combinada con el Análisis de Cluster, con el fin de analizar las concepciones de los estudiantes de secundaria sobre la importancia de la disciplina de Ciencias. en el noveno grado de la Escuela Primaria, con énfasis en conceptos de Física (Óptica). Para ello, se aplicó un cuestionario piloto, puesto a disposición virtualmente de 21 estudiantes de secundaria de un colegio público federal, ubicado en la ciudad de Campo Grande en el estado de Mato Grosso do Sul. Esta investigación es de carácter cuantitativo, en la que busca dar respuesta al objeto de estudio, caracterizándose como concluyente. La investigación requiere información definida, formal y estructurada, por lo que se presenta una muestra y el análisis de los datos es estadístico y concluyente. Al aplicar todas las pruebas y análisis (Alfa de Cronbach, Prueba de Bartlett, KMO, Análisis de 
Componentes Principales, Análisis Factorial y Análisis de Cluster), se concluye que el instrumento tiene confiabilidad, comunalidad, idoneidad e interdependencia entre las variables, siendo validado por técnicas de Análisis Multivariante.

Palabras clave: Ensenãnza; Alfa de Cronbach; Análisis factorial; Análisis de conglomerados; Física.

\section{Introdução}

O aluno do Ensino Médio (EM) se recorda das aulas de Ciências do nono ano do Ensino Fundamental (EF)? O aluno consegue empregar estes conhecimentos no seu dia a dia, a fim de tomar decisões críticas com base científica? Quais são as suas concepções a respeito do Ensino de Ciências (EC)? Qual o posicionamento do aluno frente a disciplina de Ciências, esta que articula e unifica Biologia, Física e Química? O que ele recorda referente aos conceitos do tema 'Óptica' que aborda ausência e presença da luz, difração, espelhamento, refração, reflexão e velocidade das ondas? Tais questionamentos surgem diante das inquietações das autoras por obter respostas perante a uma realidade que advém da formação inicial de professores de Ciências da Natureza.

Sendo assim, o objetivo deste estudo é descrever o processo de construção e validação de um instrumento por meio da Análise Multivariada (AM), no qual utilizou-se a técnica da Análise Fatorial (AF) combinada com a Análise de Cluster (AC), a fim de analisar as concepções dos alunos do EM acerca da importância da disciplina de Ciências do nono ano do EF, com ênfase em conceitos de Física (Óptica). Para isso, aplicou-se um questionário piloto, disponibilizado de forma virtual para 21 alunos do EM de um colégio público federal, localizado na cidade de Campo Grande do estado do Mato Grosso do Sul (MS).

Segundo Matos e Rodrigues (2019), a AM surgiu em meados da década de 1901. Contudo, a utilização da AM na área da Educação e no EC é pouco explorada, visto que as técnicas que a análise exige muitas vezes não são contempladas em sua plenitude. Ao fazer uma busca na literatura, percebe-se que a muito tempo pesquisas relacionadas com o EC são qualitativas. Oliveira (2018) ressalta que desde 1960 as pesquisas na área de EC são qualitativas e que muitas revistas científicas não aceitam artigos quantitativos na área do EC.

Greca (2002) e Oliveira (2018) ressaltam a importância da integração das pesquisas qualitativas e quantitativas, com o propósito de reduzir a distância entre os métodos. Em uma revisão da literatura (RL) sobre as metodologias adotadas em artigos de EC, Carvalho, Oliveira e Rezende (2009) constataram que de 89 artigos apenas um foi com abordagem quantitativa. Na pesquisa realizada por Schneider, Fujii e Corazza (2017), de um total de 240 artigos selecionados, nenhum adotou a abordagem quantitativa.

Para Freitas, Campos, Andrade e Régnier (2019) é necessário haver uma reflexão da importância da análise estatística, seja ela por meio da AM ou outro método adotado para comprovar pesquisas referentes ao EC. Desta forma, os autores destacam a importância de pesquisadores aplicarem corretamente as análises, uma vez que em muitos estudos a estatística não é contemplada corretamente.

A AM corresponde a vários tipos de métodos e técnicas que buscam uma interpretação teórica de um conjunto de dados (Dias, Silva e Macedo, 2019). Para tal, adotou-se a AF e a AC. De acordo com Matos e Rodrigues (2019, p.9) “a análise fatorial é utilizada para investigar os padrões ou relações latentes para um número grande de variáveis e determinar se a informação pode ser resumida a um conjunto menor de fatores”. Assim, a AF reduz o número de dimensões necessárias, por meio de fatores com a finalidade de descrever variáveis advindas dos dados coletados (Pereira, Paluto, Vieira \& Cerbaro, 2019). Tanaka, Junior, Cristo, Spedo, e Pinto (2015, p.36) conceitua a AC como sendo uma "técnica que possibilita a identificação de grupos com características homogêneas, que pode ser usada quando se tem pelo menos três variáveis numéricas". Uma das técnicas mais comumente utilizadas em clustering é k-medias, que consiste em desagregar um conjunto de objetos em subconjuntos menores, segundo suas características (variáveis).

Perante o exposto e com a necessidade de elaborar instrumentos de mensuração para o EC, com base na estatística e 
no uso de métodos e técnicas de AM, o questionário discriminado e avaliado neste estudo visa apresentar uma opção para analisar e mensurar as concepções dos alunos do EM em relação a disciplina de Ciências do EF e sobre os conceitos de Óptica relacionados à Física do nono ano. Para tal, a seguir é relatado o passo a passo da técnica adotada para a validação.

\section{Metodologia}

Esta pesquisa é de cunho quantitativo, no qual se busca responder o objeto de estudo, caracterizando-se como conclusiva, conforme Pereira et al (2018). Justificando-se por ser uma pesquisa em que as informações necessárias são definidas, formal e estruturadas; apresenta uma amostra e a análise dos dados são estatísticos e conclusivos (Marconi \& Lakatos, 2017).

Utilizou-se uma amostra probabilística aleatória com alunos que estão cursando o $3^{\circ}$ ano do EM de um colégio público federal na cidade de Campo Grande (MS), no ano de 2021. A população da turma é de 29 alunos, considerando-se um intervalo de confiança de 95\%, desvio padrão de 1,96 e um erro amostral de 0,05, tem-se que o tamanho da amostra calculada é de 21 alunos.

Para atender o objetivo da sua aplicação, bem como levar em consideração a investigação dos conceitos de EC e da Física (Óptica) relacionado ao conteúdo de Ciências do nono ano do EF, adotou-se estratégias para a elaboração do instrumento (Figura 1). Segundo Camillo (2020) um instrumento tem como finalidade auxiliar o pesquisador aprofundar-se no tema de estudo, a fim de gerar dados e respostas.

Outro aspecto importante é o delineamento da amostra (para quem elaborar/aplicar o instrumento), qual o tipo de linguagem e quais termos técnicos adotar. Busca-se neste caso ajustar ao público adolescente: a natureza das questões, que devem estar ligadas ao objeto de estudo considerando as suas classes; decidir se a ordem das questões é importante, uma vez que na literatura encontra-se a necessidade em diversificar os tipos de questões para que o participante esteja atento ao responder; e a técnica estatística adotada é fundamental, sendo que o pesquisador precisa ter um delineamento do passo a passo. Por conseguinte, é preciso haver clareza acerca de quais dados que se pretende obter nos pós coleta: como será feita a sua quantificação e qual o número de questões, sendo estas claras, objetivas e que não sejam extensas/exaustivas ao leitor. 
Figura 1. Estratégias para a elaboração de um instrumento.

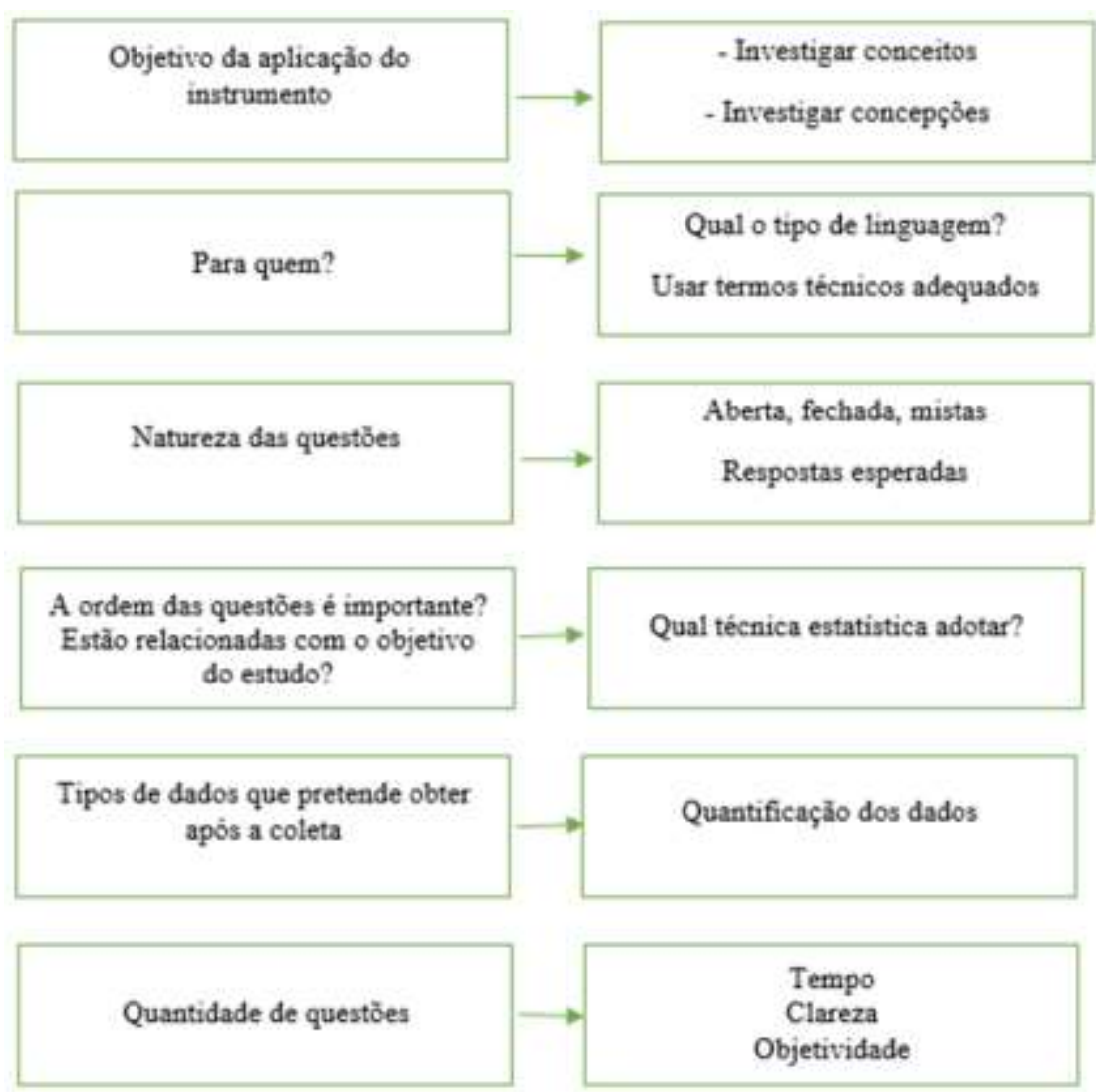

Fonte: Autoras.

O questionário foi elaborado no Google Forms e disponibilizado de forma virtual pelo Ambiente Virtual de Ensino e Aprendizagem (AVEA) do colégio. Contém 25 questões, das quais 22 são fechadas, em que se utilizou escala de escolha múltipla simples nominal e ordinal, escala de opções múltiplas simples, escala check-list, escala dicotômica simples e a escala Likert (3 e 5 pontos).

Algumas questões possuem mais de um item (Tabela 1) e adotou-se escore para cada item, conforme a Tabela 2. Segundo Damoro e Vieira (2013) escalas Likert com três pontos oferecem respostas suficientes para validação de questionário e, escalas com cinco pontos possuem um nível adequado de confiabilidade.

Tabela 1. Escala Likert adotadas para o instrumento.

\begin{tabular}{l|l|l}
\hline $\begin{array}{l}\text { Escala Likert } \\
\text { (pontos) }\end{array}$ & \multicolumn{1}{|c}{ Questões } & \multicolumn{1}{|c}{ Itens } \\
\hline 3 pontos & $\begin{array}{l}\text { Q8, Q9, Q10, Q11, Q12, Q15, } \\
\text { Q19, Q23 }\end{array}$ & $\begin{array}{l}\text { Q8 (a, b, c); Q10 (a, b, c); Q11 (a, b); } \\
\text { Q15 (a, b, c); Q19 (a, b, c, d) }\end{array}$ \\
\hline 5 pontos & Q14, Q16, Q20, Q22 & Q20 (a, b, c, d, e, f, g) \\
\hline
\end{tabular}

Fonte: Autoras. 
Tabela 2. Valores dos escores adotados para a Escala Likert de 3 e 5 pontos.

\begin{tabular}{l|c|c|c}
\hline \multirow{2}{*}{$\begin{array}{l}\text { Oscala } \\
\text { Padriginal }\end{array}$} & Valores da Escala & $\mathbf{3}$ Pontos & 5 Pontos \\
\cline { 2 - 4 } & 1 & 1 & 1 \\
\cline { 2 - 4 } & 2 & 2 & 1,5 \\
\cline { 2 - 4 } & 3 & 3 & 2,5 \\
\cline { 2 - 4 } & 4 & - & 3 \\
\cline { 2 - 4 } & 5 & - & 2 \\
\hline
\end{tabular}

Fonte: Autoras.

As questões foram agrupadas por: perfil do aluno (sete questões) e 18 questões que abordam o EC - categorizadas: aprendizagens de Ciências, argumentação, criticidade, conceitos, contextualização, relacionamento aluno-professor, comportamento, materiais didáticos e práticas didáticas. Os dados foram organizados em planilha eletrônica no programa Microsoft Excel® e analisados com auxílio do SPSS, versão 20.0®, para Windows.

Para a validação de um questionário é necessário seguir alguns passos, primeiramente é necessário padronizar as variáveis com a finalidade de que o banco de dados possua a mesma escala de valor (para este estudo foi utilizado duas escalas Likert diferentes). Para isso, é necessário calcular o Desvio Padrão (DP) e a média das variáveis, que verificam se a amostra possui uma distribuição normal. Após, é preciso testar a confiabilidade interna dos dados, aplicando-se o teste de Alfa de Cronbach.

Para a validação deste questionário, foram adotadas as sugestões de Freitas e Rodrigues (2005) para os valores de classificação da confiabilidade do coeficiente Alfa de Cronbach:

$$
\begin{array}{ll}
\text { - } & \alpha \leq 0,30-\text { Muito baixa } \\
\text { - } & 0,30<\alpha \leq 0,60-\text { Baixa } \\
\text { - } & 0,60<\alpha \leq 0,75-\text { Moderada } \\
\text { - } & 0,75<\alpha \leq 0,90 \text { - Alta } \\
\text { - } & \alpha>0,90 \text { - Muito alta }
\end{array}
$$

Posteriormente, para seguir com a validação calcula-se a comunalidade, que é um índice de variabilidade total explicada por todos os fatores para cada variável: quanto maior o valor da comunalidade, maior é o seu poder de explicação. Matos e Rodrigues (2019, p.32) afirmam que "esses métodos garantem resultados mais estáveis e mais confiáveis, que mantêm ainda o tipo de correlação existente entre os fatores, sejam eles ortogonais ou não". Ainda segundo os autores, para que se tenha uma boa comunalidade é necessário que o seu valor seja alto, acima de 0,6.

Para verificar se realmente a AF pode ser realizada, testa-se a adequabilidade através do teste KMO (Kaiser - Meyer Oklin) e o teste de esfericidade de Barlett. Segundo Matos e Rodrigues (2019, p. 43) "essa medida, que varia entre 0 e 1 , representa a proporção da variância das variáveis que pode ser explicada pelos fatores ou traços latentes". Quanto mais próximo esse valor estiver de 1, mais adequados os dados estão para se ajustar uma AF. Portanto, para que se tenha um valor de adequabilidade considerado bom, considera-se que ele seja maior de 0,8, em conformidade com Field, Miles e Field (2012). Contudo, para que o teste de Barlett tenha significância, o p-valor precisa ser menor do que o KMO.

Confirmada a significância de ambos os testes, verifica-se a interdependência por meio da análise dos componentes principais (ACP) e da AC, para finalmente completar a AF exploratória, conforme Figura 2. A ACP é utilizada para reduzir os dados para a construção de novas variáveis, técnica suficiente para validar um instrumento. Entretanto, foi aplicado a AC que “visa classificar objetos ou casos em grupos relativamente homogêneos, chamados de conglomerados" (Vicini \& Souza, 2005). 
Figura 2. Esquema dos métodos estatísticos para a validação de um questionário.

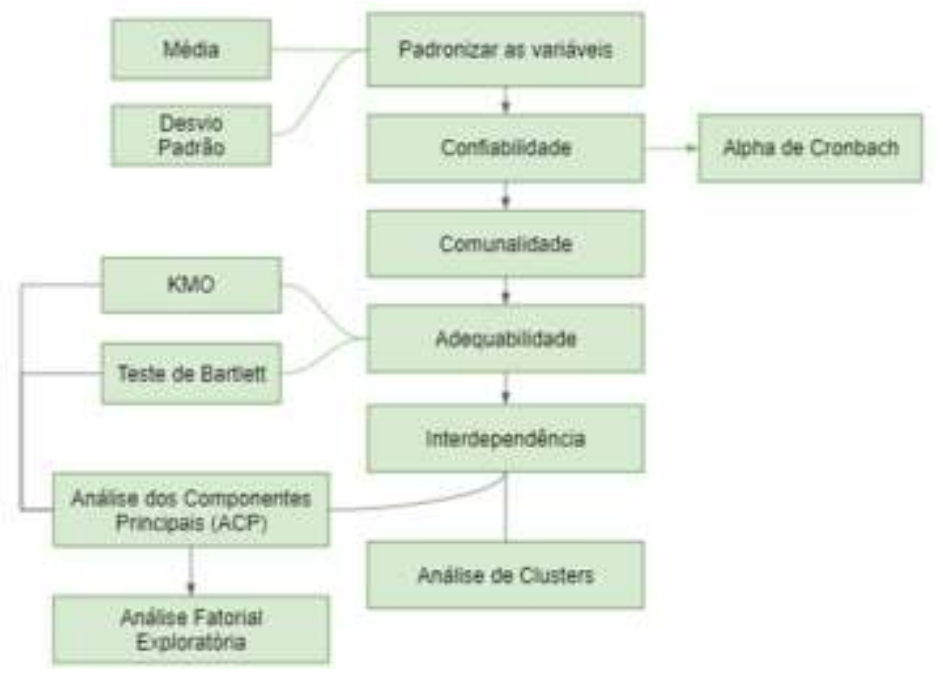

Fonte: Autoras.

\section{Resultados e Discussão}

Neste tópico será apresentado os dados referentes a validação do instrumento, seguindo os métodos estatístico, já descritos na metodologia.

\subsection{Validação do Instrumento}

A amostra contou com 21 alunos, 53\% do sexo masculino e 41,7\% do sexo feminino. Em relação à idade, $41,7 \%$ possuem 17 anos, 41,7\% têm 16 anos e 16,7\% dos alunos têm 19 anos. O questionário se mostrou dinâmico e de preenchimento rápido e didático, sendo que os alunos levaram em torno de quatro a cinco minutos para responder 25 questões.

Primeiramente realizou-se a padronização dos dados por meio da média e do desvio padrão (DP) de cada item. Neste caso, estas medidas são calculadas somente para esse fim, perante o questionário possuir duas escalas Likerts diferentes. Os dados assumem a mesma unidade, assumindo a forma (Z). Conforme Vincini e Souza (2005) a padronização remove "a influência da unidade de medida", reduzindo e descrevendo os conglomerados sem afetar o grau de similaridade.

Conforme a Figura 3, foi testado a confiabilidade de todas as variáveis em que obteve-se o valor do Alfa de Cronbach 0,751 , concluindo-se que a confiabilidade interna dos dados é alta. 
Figura 3. Teste do Alfa de Cronbach.
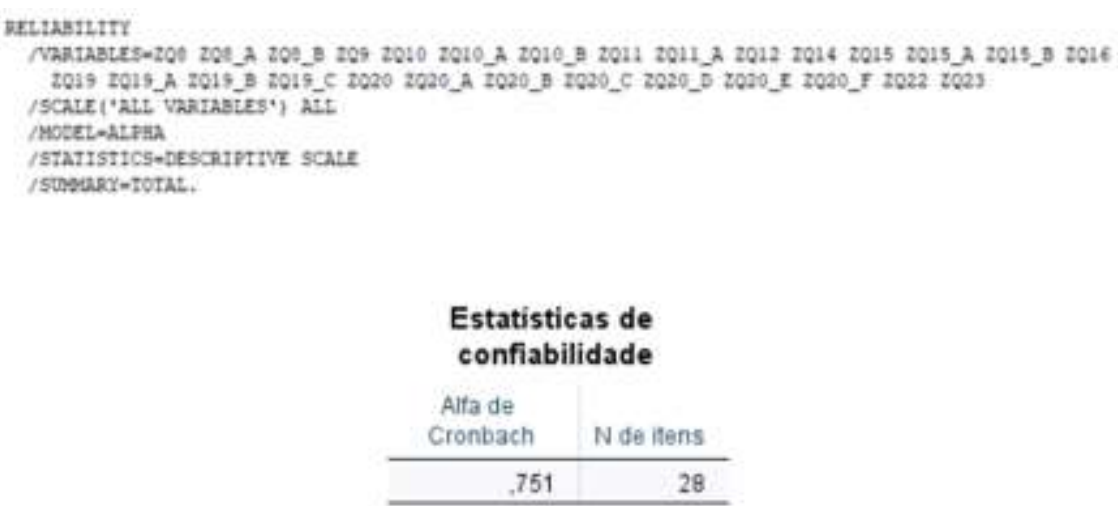

Fonte: Autoras.

Ainda sobre a confiabilidade das variáveis, o instrumento não revelou nenhum problema em relação a colinearidade, as correlações bivariadas foram menores de 0,9 e, os valores de Fator de Inflação da variância inferior a 3,0. Sendo assim, o instrumento apresenta-se com baixa multicolinearidade, podendo afirmar-se que mais informações serão aferidas pelas estimativas dos coeficientes.

Em relação a comunalidade, observa-se que todos os resultados (Tabela 3) estão acima de 0,6 o que indica que podese seguir com AF. De acordo com Schawb (2007), as comunalidades representam a proporção da variância para cada variável incluída na análise, o qual é explicada pelos componentes extraídos, por exemplo, para o item Q12, o fator extraído explica $85 \%$ da variância.

Tabela 3. Resultado das Comunalidades.

\begin{tabular}{|c|c|c|}
\hline & Inicial & $\begin{array}{r}\text { Extraçẵo } \\
871\end{array}$ \\
\hline Zscore(09) & 1,000 & .847 \\
\hline Zseore(010) & 1,000 & .865 \\
\hline Zscore 010 & 1,000 & .868 \\
\hline Zscore: 010 & 1,000 & .927 \\
\hline Zscore(011) & 1,000 &, 716 \\
\hline Zscore: 011 & 1,000 & .883 \\
\hline Zscore(012) & 1,000 & .851 \\
\hline Zscore(Q14) & 1,000 & .858 \\
\hline Zscore(015) & 1,000 & .763 \\
\hline Zscore: 016 & 1,000 & .881 \\
\hline Zscore: 015 & 1,000 & .745 \\
\hline Zscore(016) & 1,000 & .740 \\
\hline Zscore(019) & 1,000 & .741 \\
\hline Zscore 019 & 1,000 & .802 \\
\hline Zscore. 019 & 1,000 & .692 \\
\hline Zscore: 019 & 1,000 & 940 \\
\hline Zscore(020) & 1,000 & .922 \\
\hline Zscore 020 & 1,000 & .697 \\
\hline Zscore: 020 & 1,000 & .900 \\
\hline Zscore 020 & 1,000 & .848 \\
\hline Zscore: 020 & 1,000 &, 918 \\
\hline Zscore 020 & 1,000 & .763 \\
\hline Zscore: 020 & 1,000 & .941 \\
\hline Zscore(022) & 1,000 & .735 \\
\hline Zscore(Q23) & 1,000 & .938 \\
\hline
\end{tabular}

Fonte: Autoras.

Além do KMO, o teste de esfericidade de Bartlett verifica a adequabilidade da amostra. Para tal, o KMO=0,79 e o teste de Bartlett com qui-quadrado $=123,28, \mathrm{gl}=55$ e sig. $=<0,001$, conforme Tabela 4 , reforçam que o instrumento é adequado 
e os dados se ajustam para a AF.

Tabela 4. Adequabilidade da Amostra.

\section{Teste de KMO e Bartlett}

\begin{tabular}{|c|c|c|}
\hline \multicolumn{2}{|c|}{$\begin{array}{l}\text { Medida Kaiser-Meyer-Olkin de adequação de } \\
\text { amostragem. }\end{array}$} &, 787 \\
\hline \multirow{3}{*}{$\begin{array}{l}\text { Teste de Esfericidade de } \\
\text { Bartlett }\end{array}$} & Aprox. Qui-quadrado & 123,283 \\
\hline & $\mathrm{gl}$ & 55,000 \\
\hline & Sig. & $<, 001$ \\
\hline
\end{tabular}

Fonte: Autoras.

Com os dados padronizados e aferidos, e a confiabilidade, a comunalidade e a adequabilidade segue-se para a ACP, propriamente dita. Para isso, foi necessário determinar o número de fatores que se desejavam na análise. Conforme a Tabela 5, é descrito os autovalores (eigenvalue), que segundo Kaiser (1974) deve considerar os valores superiores a 1 e adotar os cinco primeiros fatores que somados a variância explicam $81,35 \%$.

Tabela 5. Números de Fatores da AF.

\begin{tabular}{|l|l|l|}
\hline $\mathrm{PC}$ & Eigenvalue & $\%$ variance \\
\hline 1 & 4,68079 & 29,327 \\
\hline 2 & 3,28801 & 20,601 \\
\hline 3 & 2,67053 & 16,732 \\
\hline 4 & 1,22953 & 7,7035 \\
\hline 5 & 1,1149 & 6,9853 \\
\hline 6 & 0,692244 & 4,3372 \\
\hline 7 & 0,607079 & 3,8036 \\
\hline 8 & 0,551449 & 3,455 \\
\hline 9 & 0,364491 & 2,2837 \\
\hline 10 & 0,252285 & 1,5807 \\
\hline 11 & 0,219579 & 1,3758 \\
\hline 12 & 0,127048 & 0,79601 \\
\hline 13 & 0,0947405 & 0,59359 \\
\hline 14 & 0,036503 & 0,22871 \\
\hline 15 & 0,0310625 & 0,19462 \\
\hline 16 & 0,000435101 & 0,0027261 \\
\hline
\end{tabular}

Fonte: Autoras.

Conforme Figueiredo e Silva (2010) e Matos e Rodrigues (2019), a escolha dos fatores da AF deve ter um ajuste em que o número de fatores seja sempre reduzido e que a soma da variância seja de $70 \%$ ou mais. Os autores ressaltam também, que não existe um único critério para definir o número de fatores ideais, sendo papel do pesquisador determinar um critério consensual. Como se deseja reduzir os dados para quatro fatores e considerar vários critérios, e um deles é que os primeiros componentes explicam $70 \%$ da variância acumulada, será adotado os quatro primeiros fatores, conforme o Gráfico 1 . O gráfico de escarpa deixa explícito que é correto considerar quatro fatores, visto que o corte sempre ocorre quando a linha sofre um desnivelamento. 
Gráfico 1. Gráfico de escarpa para definição dos fatores da AF.

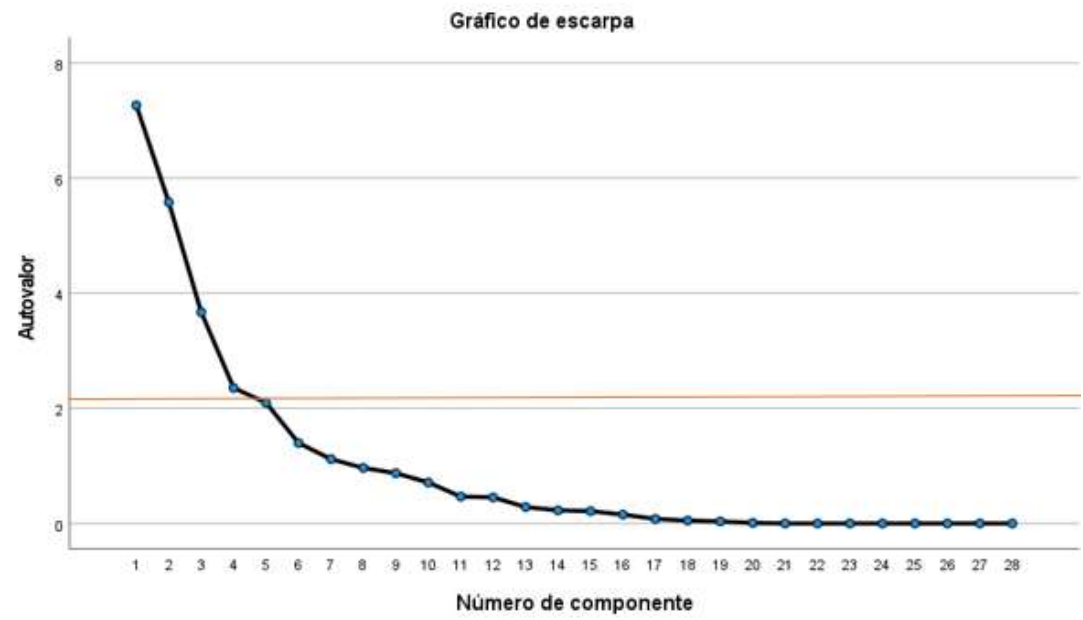

Fonte: Autoras.

Ainda, realizou-se a matriz de correlação de Pearson que apresentou significância no nível 0,01 e no nível 0,05 nas duas extremidades. Logo, prosseguindo a AF, gerou-se o dendrograma (Figura 4) onde foi verificado a formação de dois clusters, os quais possuem as variáveis de maior relevância dentro do conjunto original das variáveis do instrumento, obtidos por meio de um corte transversal feito na maior distância entre os clusters. Usou-se para o cálculo da matriz de distância o método de encadeamento único (Single Linkage), que é a distância mínima e a distância Euclidiana com ligação Ward.

Figura 4. Dendrograma com ligação de Ward.

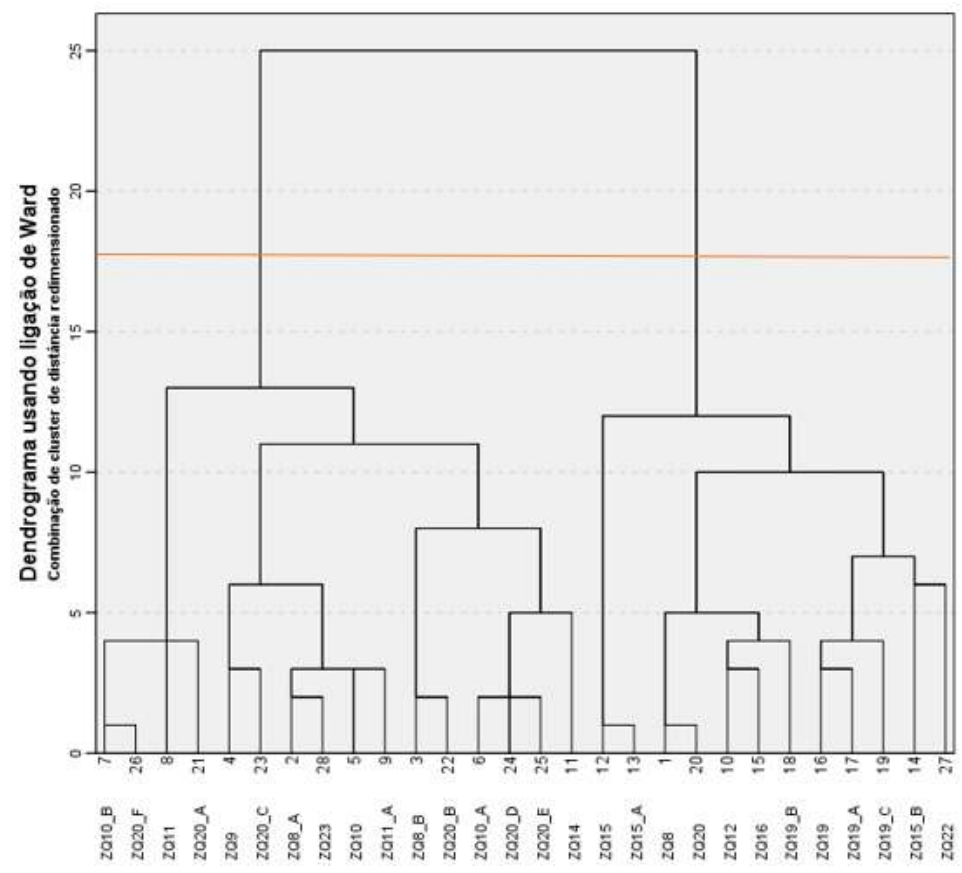

Fonte: Autoras.

Diante desse contexto, é possível afirmar que pela AC formou-se dois grupos (Quadro 1). No Grupo 1 estão os itens relacionados com o Ensino de Ciências, aprendizagem, argumentação, criticidade, conceitos e contextualização. E, no grupo 2, relacionamento aluno-professor, comportamento, materiais didáticos e práticas didáticas. 
Quadro 1. Clusters formados a partir do Dendrograma.

\begin{tabular}{|c|c|}
\hline Clusters & Itens \\
\hline $\begin{array}{l}\text { Grupo } 1 \\
\text { Cluster } 1 \text { - Relacionado ao Ensino de Ciências, } \\
\text { aprendizagem, argumentação, criticidade, } \\
\text { conceitos e contextualização. }\end{array}$ & $\begin{array}{l}\text { Q8. Motivação } \\
\text { Q9. Importância da Biologia/Física/Química } \\
\text { Q10. Capacidade de ensinar o que aprendeu } \\
\text { Q11. Ciências e a formação de opiniões } \\
\text { Q14. Entendimento do conteúdo } \\
\text { Q20. Conceitos de Física } \\
\text { Q23. Contextualização }\end{array}$ \\
\hline $\begin{array}{l}\text { Grupo } 2 \\
\text { Cluster } 2-\text { Relacionamento aluno-professor, } \\
\text { comportamento, materiais didáticos e práticas } \\
\text { didáticas. }\end{array}$ & $\begin{array}{l}\text { Q12. Experimentação/laboratório } \\
\text { Q15. Relação ao professor } \\
\text { Q16. Comportamento } \\
\text { Q19. Livro didático } \\
\text { Q.22 Aulas Práticas }\end{array}$ \\
\hline
\end{tabular}

Fonte: Autoras.

E, para finalizar, gerou-se o gráfico de componentes em espaço rotacionado (Gráfico 2). O método utilizado foi o Varimax, pois ele minimiza as cargas dentro dos fatores. Matos e Rodrigues (2019, p.38) afirmam que o método Varimax "tenta carregar um número menor de variáveis altamente em cada fator, o que resulta em grupos de fatores mais interpretáveis". Ainda, segundo Field et al. (2012), o método é o mais utilizado na AF por ser mais simples de entender e interpretar.

Gráfico 2. Gráfico de componentes em espaço rotacionado.

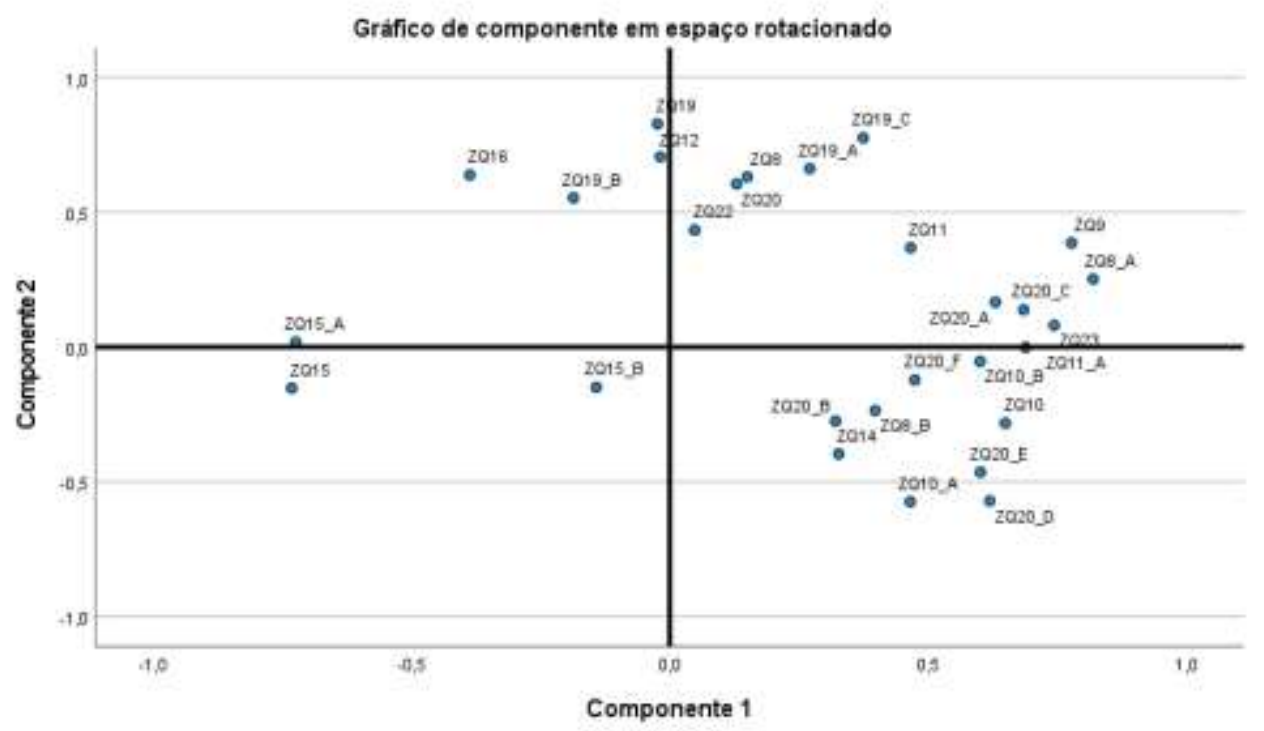

Fonte: Autoras.

Conforme Camillo, Souza e Ramser (2020) é importante que nenhuma variável esteja sobreposta a outra, a fim de que não possuam a mesma representatividade. A partir do Gráfico 2, verifica-se que as variáveis não estão sobrepostas. Nota-se que os agrupamentos são semelhantes aos formados na AC, afirmando a importância dos itens nesta validação. Outro fato importante é que nenhuma variável está fora do limite da distribuição normal em que a média é zero e o DP igual a 1.

Outro ponto relevante é que são mais de dois fatores, indicando que eles não podem ocupar o mesmo espaço. O grupo maior das variáveis estão no quadrante do lado direito/superior indicando que são os melhores itens (questões) do instrumento, do lado direito/inferior os itens são significantes, porém com menor valor. Contudo, estas variáveis estão perto do eixo x, 
aumentando o seu nível de significância na amostra. O mesmo acontece com Q15, Q15A e Q15B, são variáveis em cima do eixo x, mesmo estando em outros quadrantes estas questões possuem um nível alto de significância.

\section{Considerações Finais}

O instrumento é validado tanto em relação ao conteúdo como à estrutura interna. A construção e validação de novas ferramentas para pesquisas na área de Ciências Naturais com ênfase no EF, na Educação Básica, é de extrema importância. Existem poucos estudos com validação estatística que utilizam técnicas $\mathrm{AF}$ e a $\mathrm{AC}$, e que procedem com todos os requisitos necessários de consistência interna.

Pela AF foi mensurada a dimensionalidade de todos os itens do questionário, ou seja, mostrou-se que cada questão é importante e significante para que o pesquisador mensure as concepções do aluno referente ao Ensino de Ciências do nono ano do EF. Além disso, é possível mensurar o conhecimento a respeito dos conceitos de Óptica relacionados à Física, da disciplina de Ciências. Para tal, o Alfa de Cronbach, o KMO, o teste de esfericidade de Barlett apresentaram-se significativos, além da $\mathrm{ACP}$ e da AC.

Por fim, instrumentos como este possibilitam analisar e mensurar as concepções dos alunos em uma disciplina tão importante como a de Ciências do nono ano do EF, visto que, é uma disciplina que abrange muitos conceitos, leis e fórmulas de outras três disciplinas (Biologia, Física e Química). Destaca-se a necessidade da realização de outras pesquisas que utilizem técnicas de estatísticas em outras disciplinas, ou até mesmo avaliar alguns conceitos específicos, como foi o caso deste instrumento, que deu ênfase aos conceitos de Física (Óptica).

\section{Referências}

Camillo, C. M. (2020). Concepções dos estudantesde uma escola do campo sobre tecnologias digitais de informação e comunicação. Research, Society and Development, 9(4), e148943006, http://dx.doi.org/10.33448/rsd-v9i4.3006.

Camillo, C. M., Souza, A. \& Ramser, C. (2020). Variáveis climáticas relacionadas à poluição do ar e os efeitos causados à saúde humana. Ciência e Natura. 42. https://doi.org/10.5902/2179460X39722

Carvalho, R. C. D., Oliveira, I., \& Rezende, F. (2009). Tendências da pesquisa na área de educação em ciências: uma análise preliminar da publicação da ABRAPEC. VII Encontro Nacional de Pesquisa em Educação em Ciências, 1-12.

Dalmoro, M. \& Vieira, K. M. (2013). Dilemas na construção de escalas tipo Likert: o número de itens e a disposição influenciam os resultados. Revista Gestão Organizacional. v.6.

Dias, P., Silva. H. \& Macedo, R. (2019). Estatísticas Multivariadas na Administração: importância na aplicação e aplicação da análise fatorial exploratória. Revista Eletrônica de Administração e Turismo. 13(1).

Field, A., Miles, A. \& Field, Z. (2012). Discovering statistics using R. Sage Publications.

Figueiredo, D. B. \& Silva, J. A. (2010). Visão além do alcance: uma introdução à análise fatorial. Opinião Pública, Campinas, v. 16 , n. 1.

Freitas, A. L. P. \& Rodrigues, S. (2005). Avaliação da confiabilidade de questionário: uma análise utilizando o coeficiente alfa de Cronbach In: Simpósio De Engenharia De Produção, 12, Anais... Bauru-SP: UNESP.

Freitas, 1., Campos, A., Andrade, V. \& Régnier, J. (2019). Análise estatística implicativa da tendência de abordagens do método de estudo de casos no ensino de ciências. Amazônia: Revista de Educação em Ciências e Matemáticas. 15(11).

Greca, I. M. (2002). Discutindo aspectos metodológicos da pesquisa em ensino de ciências: algumas questões para refletir. Revista Brasileira de Pesquisa em Educação em Ciências, 2(1), 73-82.

Kaiser, H. F. (1974). An index of factorial simplicity. Psychometrika, 39, 31-36.

Marconi, M. A. \& Lakatos, E. M. (2017). Fundamentos de metodologia científica. (8a ed.), Atlas.

Matos, D. A. \& Rodrigues, E. (2019). Análise Fatorial. ENAP - Enap Fundação Escola Nacional de Administração Pública.

Medeiros, A. (2002). Metodologia da pesquisa em educação em ciências. Revista Brasileira de Pesquisa em Educação em Ciências, 2(1). 66-72.

Oliveira, G. J. (2018). Análise dos artigos da revista eletrônicas em ensino de ciências que apresentem em seu título as palavras ensino de ciências. Dissertação (Mestrado). Unipampa, Uruguaiana. RS. 
Research, Society and Development, v. 10, n. 6, e52510616149, 2021

(CC BY 4.0) | ISSN 2525-3409 | DOI: http://dx.doi.org/10.33448/rsd-v10i6.16149

Pereira, A., Paluto, B., Vieira, M. \& Cerbaro, R. (2019). Análise Fatorial. (2a ed.), Texto para Discussão.

Pereira, A. S. et al. (2018). Metodologia da pesquisa científica. UFSM.

Schawb, A.J. (2007). Eletronic classroom. Online].

Schneider, E. M., Fujii, R. A. X., \& Corazza, M. J. (2017). Pesquisas quali-quantitativas: contribuições para a pesquisa em ensino de ciências. Revista Pesquisa Qualitativa, 5(9), 569-584.

Tanaka, O. Y., Junior, M, Cristo, E, Spedo, S. M. \& Pinto, N. R. (2015). Uso da análise de clusters como ferramenta de apoio à gestão no SUS. Saúde Soc. São Paulo, 24(1), 34-45.

Vicini, L. \& Souza, A. (2005). Análise Multivariada da Teoria à Prática. TCC (Especialização em Estatística e Modelagem Quantitativa). Universidade Federal de Santa Maria. RS. 